\title{
UPPER BOUNDS FOR THE NUMBER OF ZEROES FOR SOME ABELIAN INTEGRALS
}

\author{
ARMENGOL GASULL, J. TOMÁS LÁZARO, AND JOAN TORREGROSA
}

\begin{abstract}
Consider the vector field $x^{\prime}=-y G(x, y), y^{\prime}=x G(x, y)$, where the set of critical points $\{G(x, y)=0\}$ is formed by $K$ straight lines, not passing through the origin and parallel to one or two orthogonal directions. We perturb it with a general polynomial perturbation of degree $n$ and study which is the maximum number of limit cycles that can bifurcate from the period annulus of the origin in terms of $K$ and $n$. Our approach is based on the explicit computation of the Abelian integral that controls the bifurcation and in a new result for bounding the number of zeroes of a certain family of real functions. When we apply our results for $K \leq 4$ we recover or improve some results obtained in several previous works.
\end{abstract}

\section{INTRODUCTION}

The problem of determining the number of limit cycles bifurcating from the period annulus of a system

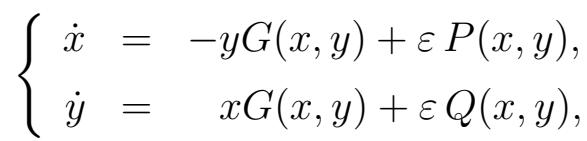

where $P(x, y), Q(x, y)$ are polynomials of a given degree, $G(x, y)$ satisfies $G(0,0) \neq 0$ and $\varepsilon$ is a small parameter, has been widely studied (see for instance $[2,4,7,8,9,10,12,13]$ ). Among this type of systems we will be concerned with those having

$$
G(x, y)=\prod_{j=1}^{K_{1}}\left(x-\mathrm{a}_{j}\right) \prod_{\ell=1}^{K_{2}}\left(y-\mathrm{b}_{\ell}\right),
$$

where $\mathrm{a}_{j}$ and $\mathrm{b}_{\ell}$ are real numbers with $\mathrm{a}_{i} \neq \mathrm{a}_{j}$ and $\mathrm{b}_{i} \neq \mathrm{b}_{j}$ for $i \neq j$. The unperturbed system $(\varepsilon=0)$ presents a centre at the origin and any line $x=\mathrm{a}_{j}$ or $y=\mathrm{b}_{\ell}$ constitutes an invariant set of singular points of the system. This invariant set is formed by parallel and/or orthogonal invariant lines.

The aim of this work is to provide, for small values of $\varepsilon$, upper bounds for the number of limit cycles bifurcating from periodic orbits of the unperturbed system in the period

2010 Mathematics Subject Classification. Primary: 34C08. Secondary: 34C07, 34C23, 37C27, $41 \mathrm{~A} 50$.

Key words and phrases. Abelian integrals, Weak 16th Hilbert's Problem, limit cycles, Chebyshev system, number of zeroes of real functions.

The first and third authors are partially supported by the MICIIN/FEDER grant number MTM200803437 and by the Generalitat de Catalunya grant number 2009SGR410. The second author is partially supported by the MICIIN/FEDER grant number MTM2009-06973 and by the Generalitat de Catalunya grant number 2009SGR859. 
annulus

$$
\mathcal{D}=\left\{(x, y) \in \mathbb{R}^{2} \mid 0<\sqrt{x^{2}+y^{2}}<\rho:=\min _{j, \ell}\left\{\left|\mathrm{a}_{j}\right|,\left|\mathrm{b}_{\ell}\right|\right\}\right\} .
$$

Several previous works handle this problem for different particular choices of small values of $K_{1}$ and $K_{2}$. The following cases have been studied: one line in [10]; two parallel lines in [13]; two orthogonal lines in [2]; three lines, two of them parallel and one perpendicular in [5]; and four lines with a special configuration in [1]. Other related works are [8], with $G(x, y)$ any quadratic polynomial; [12], one multiple singular line; or [7] for $K$ isolated singular points.

As it is standard in this type of problems, we rewrite our system (1) in the equivalent form on $\mathcal{D}$,

$$
\left\{\begin{array}{l}
\dot{x}=-y+\varepsilon P(x, y) / G(x, y), \\
\dot{y}=x+\varepsilon Q(x, y) / G(x, y),
\end{array}\right.
$$

where $G(x, y)$ is defined in (2). Let us denote by $\gamma_{r}=\left\{(x, y) \mid x^{2}+y^{2}=r^{2}\right\}$, with $0<$ $r<\rho$, any periodic orbit of the unperturbed system. It is well known that the associated return map, whose isolated zeroes give rise to limit cycles, is $\Pi(r, \varepsilon)=r+I(r) \varepsilon+\mathcal{O}\left(\varepsilon^{2}\right)$ where $I(r)$ is the Abelian integral

$$
I(r)=\int_{\gamma_{r}} \frac{Q(x, y) d x-P(x, y) d y}{G(x, y)} .
$$

This integral is the so-called (first-order) Poincaré-Melnikov-Pontryagin function. The return map $\Pi(r, \varepsilon)$ is analytic in $r$ ranging on any compact subset of $(0, \rho)$ for $\varepsilon$ small enough. It is known (see for instance [3]) that, provided $I(r)$ does not vanish exactly, the number of zeroes of $\Pi(r, \varepsilon)$, for $\varepsilon$ small enough, is at most the number of zeroes of $I(r)$ taking into account their multiplicity. The problem of estimating this number in terms of the involved degrees is commonly called the weakened Hilbert's 16th Problem.

The goal of this paper is to provide an upper bound for the number of zeroes of the Abelian integral associated to a system of the form (1), depending on the number of critical straight lines and the degree $n$ of the perturbative polynomials $P(x, y)$ and $Q(x, y)$. We prove:

Theorem 1.1. Consider a system of the form (1),

$$
\left\{\begin{array}{llr}
\dot{x} & = & -y G(x, y)+\varepsilon P(x, y), \\
\dot{y} & =x G(x, y)+\varepsilon Q(x, y),
\end{array}\right.
$$

where

$$
G(x, y)=\prod_{j=1}^{K_{1}}\left(x-\mathrm{a}_{j}\right) \prod_{\ell=1}^{K_{2}}\left(y-\mathrm{b}_{\ell}\right),
$$

$P(x, y), Q(x, y)$ are polynomials of degree $n, \mathrm{a}_{j}$ and $\mathrm{b}_{\ell}$ are real numbers with $\mathrm{a}_{i} \neq \mathrm{a}_{j}$ and $\mathrm{b}_{i} \neq \mathrm{b}_{j}$ for $i \neq j, \varepsilon$ is a small parameter and $K_{1} \geq K_{2} \geq 0$. Moreover, when $K_{2}=0$, $K_{1} \geq 1$ and $\prod_{\ell=1}^{0}\left(y-\mathrm{b}_{\ell}\right):=1$. 
Let $I(r)$ be its associated Abelian integral defined in (4). Then, the number of real zeroes of $I(r)$ in $\left(0, \min \left\{\left|\mathrm{a}_{j}\right|,\left|\mathrm{b}_{\ell}\right|\right\}\right)$, counting their multiplicities, $\mathcal{Z}(I)$, satisfies

$$
\mathcal{Z}(I) \leq \begin{cases}\widetilde{K}_{1}\left[\frac{n+3}{2}\right]+\left[\frac{n}{2}\right], & K_{2}=0 \\ \left(\widetilde{K}_{1}+\widetilde{K}_{2}\right)\left(\left[\frac{n+2}{2}\right]+L\right)+\left[\frac{n-1}{2}\right]+L, & K_{2} \geq 1\end{cases}
$$

where

$$
\begin{aligned}
\widetilde{K}_{1} & =\operatorname{Card}\left\{\left|\mathrm{a}_{1}\right|, \ldots,\left|\mathrm{a}_{K_{1}}\right|\right\} \leq K_{1}, \\
\widetilde{K}_{2} & =\operatorname{Card}\left\{\left|\mathrm{a}_{1}\right|, \ldots,\left|\mathrm{a}_{K_{1}}\right|,\left|\mathrm{b}_{1}\right|, \ldots,\left|\mathrm{b}_{K_{2}}\right|\right\}-\widetilde{K}_{1} \leq K_{2}, \\
L & =\operatorname{Card}\left\{\mathrm{a}_{j}^{2}+\mathrm{b}_{\ell}^{2}, j=1, \ldots, K_{1}, \ell=1, \ldots, K_{2}\right\} \leq K_{1} K_{2}
\end{aligned}
$$

and $[s]$ denotes the integer part of $s$.

Note that the symmetric situation, $K_{2} \geq K_{1}$, evolves in a completely similar way changing $(x, y)$ by $(y, x)$.

Recall that using this approach we know that the total number of limit cycles (counting their multiplicities) of system (1) which bifurcate from its periodic orbits is bounded by the maximum number of isolated zeroes (counting their multiplicities) of $I(r)$ for $0<r<\rho=\min \left\{\left|\mathrm{a}_{j}\right|,\left|\mathrm{b}_{\ell}\right|\right\}$.

The proof of Theorem 1.1 is based on two steps: a first one where the corresponding Abelian integral (4) is explicitly computed and a second one where an upper estimate on the number of its zeroes is provided. This is done in Section 2 and 4, respectively. This second part is supported on the following result, proved in Section 3, that we believe it is interesting by itself.

Theorem 1.2. Consider a function of the form

$$
F(x)=P^{0}(x)+\sum_{j=1}^{K} P^{j}(x) \frac{1}{\sqrt{x+c_{j}}},
$$

where $P^{j}(x), j=0, \ldots, K$, are real polynomials and $c_{j}, j=1, \ldots, K$, are real constants. Then its number of real zeroes, taking into account their multiplicities, $\mathcal{Z}(F)$, satisfies

$$
\mathcal{Z}(F) \leq K\left(\max _{j=1, \ldots, K}\left(\operatorname{deg}\left(P^{j}\right)\right)+1\right)+\operatorname{deg}\left(P^{0}\right) .
$$

Here $\operatorname{deg}(0)=-1$

In the forthcoming paper [6] the above result is extended to a wider family of functions, studying in particular its sharpness and its relation with the theory of Chebyshev systems. We only comment here that when $\operatorname{deg}\left(P^{j}\right)$ coincide for all $j=1, \ldots, K$ it can be seen that the result is sharp.

In Section 5 we apply Theorem 1.1 to some particular cases, already studied by other authors, all satisfying $K_{1}+K_{2} \leq 4$. More concretely, in that cases we show that our theorem either gives new proofs or improve the known results for the upper bounds for the number of zeroes of $I(r)$.

The main differences between our work and the previous ones are: 
- We manage to study the case of having an arbitrary number $K$ of lines of critical points for the unperturbed system. All previous results consider at most 4 lines with several relative positions.

- We prove a result (Theorem 1.2) for bounding the number of zeroes of a special type of functions which are precisely the ones that appear in the final expression of the Abelian integral $I(r)$. The previous works apply general methods like, squaring the equation to eliminate radicals, or the principle of the argument, extending the function to $\mathbb{C}$. It can be seen that when there are more than two square roots in the expression (5), Theorem 1.2 is sharper than those general methods, see [6]. This is the reason for which we can improve some of the previously given upper bounds for $\mathcal{Z}(I)$.

Some comments about the sharpness of the upper bounds provided by Theorem 1.1 are given at the end of Section 4.

\section{Explicit COMPutation of the Abelian integral}

The aim of this section is to obtain an explicit expression of the Abelian integral presented in the introduction. The first two lemmas deal with the cases of one and two perpendicular singular lines. They are already known, see $[2,10]$, but the proof that we present is shorter. The next two results extend them to the case of an arbitrary number of parallel or perpendicular lines.

Lemma 2.1. Let a be a non-zero real number. For any $0<r<|\mathrm{a}|$ and any polynomial $R_{n+1}(x, y)$ of degree $n+1$, define

$$
I_{n+1}^{\mathrm{a}}(r)=\int_{0}^{2 \pi} \frac{R_{n+1}(r \cos \theta, r \sin \theta)}{r \cos \theta-\mathrm{a}} d \theta .
$$

Then, for $n \geq 0$, one has

$$
I_{n+1}^{\mathrm{a}}(r)=\frac{S_{[(n-1) / 2]+1}\left(r^{2}\right)}{\sqrt{\mathrm{a}^{2}-r^{2}}}+T_{[n / 2]}\left(r^{2}\right),
$$

for suitable polynomials $S_{s}(\rho)$ and $T_{s}(\rho)$ of degree s. Moreover $I_{0}^{\mathrm{a}}(r)=-2 \pi R_{0} / \sqrt{\mathrm{a}^{2}-r^{2}}$.

Proof. It is easy to check that

$$
\int_{0}^{2 \pi} \frac{1}{r \cos \theta-a} d \theta=-2 \pi \frac{1}{\sqrt{a^{2}-r^{2}}} .
$$

Hence the expression for $I_{0}^{\mathrm{a}}(r)$ follows.

Let us now deal with $n \geq 0$. We will proceed inductively. When $n=0$ we have that

$$
I_{1}^{\mathrm{a}}(r)=\int_{0}^{2 \pi} \frac{a_{0,0}+a_{0,1} r \cos \theta+a_{1,0} r \sin \theta}{r \cos \theta-\mathrm{a}} d \theta .
$$


For this case we can write

$$
\begin{aligned}
I_{1}^{\mathrm{a}}(r)= & I_{0}^{\mathrm{a}}(r)+a_{0,1} \int_{0}^{2 \pi} \frac{r \cos \theta}{r \cos \theta-\mathrm{a}} d \theta+a_{1,0} \int_{0}^{2 \pi} \frac{r \sin \theta}{r \cos \theta-\mathrm{a}} d \theta= \\
& I_{0}^{\mathrm{a}}(r)+a_{0,1} \int_{0}^{2 \pi} \frac{r \cos \theta-\mathrm{a}+\mathrm{a}}{r \cos \theta-\mathrm{a}} d \theta=I_{0}^{\mathrm{a}}(r)+2 \pi a_{0,1}+a_{0,1} \mathrm{a} \int_{0}^{2 \pi} \frac{1}{r \cos \theta-\mathrm{a}} d \theta= \\
& 2 \pi a_{0,1}-2 \pi\left(a_{0,0}+a_{0,1} \mathrm{a}\right) \frac{1}{\sqrt{\mathrm{a}^{2}-r^{2}}} .
\end{aligned}
$$

Now we will prove the expression for $I_{n+1}^{\mathrm{a}}(r)$ by induction on the degree. By hypothesis of induction it is enough to prove the formula when $R_{n+1}(x, y)$ is a homogeneous polynomial of degree $n+1$. If we write

$$
\int_{0}^{2 \pi} \frac{\sum_{i=0}^{n+1} r^{n+1} a_{i, n+1-i} \sin ^{i} \theta \cos ^{n+1-i} \theta}{r \cos \theta-\mathrm{a}} d \theta=r^{n+1} \sum_{i=0}^{n+1} \int_{0}^{2 \pi} \frac{a_{i, n+1-i} \sin ^{i} \theta \cos ^{n+1-i} \theta}{r \cos \theta-\mathrm{a}} d \theta
$$

using symmetry properties of the integrated functions, we know that all the integrals with an odd exponent in $\sin \theta$ are zero. Then we have

$$
\begin{aligned}
& r^{n+1} \sum_{j=0}^{[(n+1) / 2]} a_{2 j, n+1-2 j} \int_{0}^{2 \pi} \frac{\sin ^{2 j} \theta \cos ^{n+1-2 j} \theta}{r \cos \theta-\mathrm{a}} d \theta= \\
& r^{n+1} \sum_{j=0}^{[(n+1) / 2]} a_{2 j, n+1-2 j} \int_{0}^{2 \pi} \frac{\left(1-\cos ^{2} \theta\right)^{j} \cos ^{n+1-2 j} \theta}{r \cos \theta-\mathrm{a}} d \theta= \\
& \sum_{j=0}^{[(n+1) / 2]} a_{2 j, n+1-2 j} \sum_{k=0}^{j} r^{2 j-2 k}(-1)^{j}\left(\begin{array}{l}
j \\
k
\end{array}\right) \int_{0}^{2 \pi} \frac{r^{n+1+2 k-2 j} \cos ^{n+1-2 j+2 k} \theta}{r \cos \theta-\mathrm{a}} d \theta .
\end{aligned}
$$

When $k<j$ we can use again the induction hypotheses for each term of the sum and the statement is proved because when we multiply by the monomial $\left(r^{2}\right)^{j-k}$, the degrees, in $r^{2}$, of the polynomials $S\left(r^{2}\right)$ and $T\left(r^{2}\right)$ are $([(n+2 k-2 j-1) / 2]+1)+(j-k)=$ $[(n-1) / 2]+1$ and $[(n+2 k-2 j) / 2]+(j-k)=[n / 2]$, respectively.

The unique case that remains to check is $k=j$. For it we can write

$$
\begin{aligned}
\int_{0}^{2 \pi} \frac{r^{n+1} \cos ^{n+1} \theta}{r \cos \theta-\mathrm{a}} d \theta= & \int_{0}^{2 \pi} \frac{r^{n} \cos ^{n} \theta(r \cos \theta-\mathrm{a}+\mathrm{a})}{r \cos \theta-\mathrm{a}} d \theta= \\
& \int_{0}^{2 \pi} r^{n} \cos ^{n} \theta d \theta+\mathrm{a} \int_{0}^{2 \pi} \frac{r^{n} \cos ^{n} \theta}{r \cos \theta-\mathrm{a}} d \theta .
\end{aligned}
$$

We will treat separately the cases $n$ even and $n$ odd.

When $n$ is even the first term of the above sum is a polynomial of degree $n / 2$ in $r^{2}$ and for the second term, using the induction hypothesis, the polynomials $S\left(r^{2}\right)$ and $T\left(r^{2}\right)$ are of degree $[((n-1)-1) / 2]+1=(n-2) / 2+1=n / 2=[(n-1) / 2]+1$ and $[(n-1) / 2]=(n-2) / 2$, respectively. The statement is proved, in this case, because we should add the monomial of degree $n / 2$ corresponding to the first summand. Hence the degree of the polynomial $T\left(r^{2}\right)$ is $n / 2=[n / 2]$. 
When $n$ is odd the first term in the sum is zero. For the second term, from the induction hypothesis, the polynomials $S\left(r^{2}\right)$ and $T\left(r^{2}\right)$ are of degree $[((n-1)-1) / 2]+1=$ $(n-3) / 2+1=(n-1) / 2=[(n-1) / 2]$ and $[(n-1) / 2]=[n / 2]$, respectively. Note that the contribution of this term to the polynomial $S\left(r^{2}\right)$ has one degree less than expected but the total degree is $[(n-1) / 2]+1$ because it appears for the other terms. More concretely in the $(\sin \theta)^{[(n+1) / 2]}$ term.

Lemma 2.2. Let $\mathrm{a}$ and $\mathrm{b}$ be non-zero real numbers. For any $0<r<\min (|\mathrm{a}|,|\mathrm{b}|)$ and any polynomial $R_{n+1}(x, y)$ of degree $n+1$ consider

$$
I_{n+1}^{\mathrm{a}, \mathrm{b}}(r)=\int_{0}^{2 \pi} \frac{R_{n+1}(r \cos \theta, r \sin \theta)}{(r \cos \theta-\mathrm{a})(r \sin \theta-\mathrm{b})} d \theta .
$$

Then, for $n \geq 0$, we have that

$$
I_{n+1}^{\mathrm{a}, \mathrm{b}}(r)=\frac{1}{\mathrm{a}^{2}+\mathrm{b}^{2}-r^{2}}\left(\frac{U_{[n / 2]+1}\left(r^{2}\right)}{\sqrt{\mathrm{a}^{2}-r^{2}}}+\frac{V_{[n / 2]+1}\left(r^{2}\right)}{\sqrt{\mathrm{b}^{2}-r^{2}}}\right)+W_{[(n-1) / 2]}\left(r^{2}\right),
$$

for some given polynomials $U_{s}(\rho), V_{s}(\rho)$ and $W_{s}(\rho)$ of degree s. Moreover

$$
I_{0}^{\mathrm{a}, \mathrm{b}}(r)=2 \pi \frac{R_{0}}{\mathrm{a}^{2}+\mathrm{b}^{2}-r^{2}}\left(\frac{\mathrm{a}}{\sqrt{\mathrm{a}^{2}-r^{2}}}+\frac{\mathrm{b}}{\sqrt{\mathrm{b}^{2}-r^{2}}}\right) .
$$

Proof. Applying for instance residues formula, we obtain

$$
\int_{0}^{2 \pi} \frac{d \theta}{(r \cos \theta-a)(r \sin \theta-b)}=\frac{2 \pi}{a^{2}+b^{2}-r^{2}}\left(\frac{a}{\sqrt{a^{2}-r^{2}}}+\frac{b}{\sqrt{b^{2}-r^{2}}}\right) .
$$

Therefore the expression for $I_{0}^{\mathrm{a}, \mathrm{b}}(r)$ follows.

To obtain the result for all $n$ we will proceed inductively. When $n=0$ we have that

$$
I_{1}^{\mathrm{a}, \mathrm{b}}(r)=\int_{0}^{2 \pi} \frac{a_{0,0}+a_{0,1} r \cos \theta+a_{1,0} r \sin \theta}{(r \cos \theta-\mathrm{a})(r \sin \theta-\mathrm{b})} d \theta .
$$

For this case we can write

$$
\begin{aligned}
I_{1}^{\mathrm{a}, \mathrm{b}}(r)= & I_{0}^{\mathrm{a}, \mathrm{b}}(r)+a_{0,1} \int_{0}^{2 \pi} \frac{r \cos \theta-\mathrm{a}+\mathrm{a}}{(r \cos \theta-\mathrm{a})(r \sin \theta-\mathrm{b})} d \theta+ \\
& a_{1,0} \int_{0}^{2 \pi} \frac{r \sin \theta-\mathrm{b}+\mathrm{b}}{(r \cos \theta-\mathrm{a})(r \sin \theta-\mathrm{b})} d \theta=I_{0}^{\mathrm{a}, \mathrm{b}}(r)+a_{0,1} \int_{0}^{2 \pi} \frac{1}{r \sin \theta-\mathrm{b}} d \theta+ \\
& a_{1,0} \int_{0}^{2 \pi} \frac{1}{r \cos \theta-\mathrm{a}} d \theta+\left(a_{0,1} \mathrm{a}+a_{1,0} \mathrm{~b}\right) \int_{0}^{2 \pi} \frac{1}{(r \cos \theta-\mathrm{a})(r \sin \theta-\mathrm{b})} d \theta= \\
& a_{0,1} \frac{-2 \pi}{\sqrt{\mathrm{b}^{2}-r^{2}}}+a_{1,0} \frac{-2 \pi}{\sqrt{\mathrm{a}^{2}-r^{2}}}+\frac{a_{0,0}+a_{0,1} \mathrm{a}+a_{1,0} \mathrm{~b}}{\mathrm{a}^{2}+\mathrm{b}^{2}-r^{2}}\left(\frac{2 \pi \mathrm{a}}{\sqrt{\mathrm{b}^{2}-r^{2}}}+\frac{2 \pi \mathrm{b}}{\sqrt{\mathrm{a}^{2}-r^{2}}}\right) .
\end{aligned}
$$

This last expression satisfies the statement with $U\left(r^{2}\right)$ and $V\left(r^{2}\right)$ polynomials of degree $[n / 2]+1=[0 / 2]+1=1$ in $r^{2}$ and the polynomial $W\left(r^{2}\right)$ is identically zero.

Now we will prove the expression for $I_{n+1}^{\mathrm{a}, \mathrm{b}}(r)$ by induction on the degree of $R_{n}(x, y)$. As in the proof of Lemma 2.1, by induction hypothesis, it is enough to prove the result 
when $R_{n+1}(x, y)$ is a homogeneous polynomial of degree $n+1$. For this case we can write

$$
\begin{aligned}
& \int_{0}^{2 \pi} \frac{\sum_{i=0}^{n+1} r^{n+1} a_{i, n+1-i} \sin ^{i} \theta \cos ^{n+1-i} \theta}{(r \cos \theta-\mathrm{a})(r \sin \theta-\mathrm{b})} d \theta=\sum_{i=0}^{n+1} \int_{0}^{2 \pi} \frac{r^{n+1} a_{i, n+1-i} \sin ^{i} \theta \cos ^{n+1-i} \theta}{(r \cos \theta-\mathrm{a})(r \sin \theta-\mathrm{b})} d \theta= \\
& \sum_{j=0}^{\left[\frac{n}{2}\right]} \int_{0}^{2 \pi} \frac{r^{n+1} a_{2 j+1, n+1-(2 j+1)} \sin \theta\left(1-\cos ^{2} \theta\right)^{j} \cos ^{n+1-(2 j+1)} \theta}{(r \cos \theta-\mathrm{a})(r \sin \theta-\mathrm{b})} d \theta+ \\
& \sum_{j=0}^{\left[\frac{n+1}{2}\right]} \int_{0}^{2 \pi} \frac{r^{n+1} a_{2 j, n+1-2 j}\left(1-\cos ^{2} \theta\right)^{j} \cos ^{n+1-2 j} \theta}{(r \cos \theta-\mathrm{a})(r \sin \theta-\mathrm{b})} d \theta= \\
& \sum_{j=0}^{\left[\frac{n}{2}\right]} \sum_{k=0}^{j}\left(\begin{array}{l}
j \\
k
\end{array}\right)(-1)^{k} a_{2 j+1, n-2 j} r^{2(j-k)} \int_{0}^{2 \pi} \frac{r^{n-2(j-k)+1} \sin \theta \cos ^{n-2(j-k)} \theta}{(r \cos \theta-\mathrm{a})(r \sin \theta-\mathrm{b})} d \theta+ \\
& {\left[\frac{n+1}{2}\right]} \\
& \sum_{j=0}^{j} \sum_{k=0}^{j}\left(\begin{array}{l}
j \\
k
\end{array}\right)(-1)^{k} a_{2 j, n+1-2 j} r^{2(j-k)} \int_{0}^{2 \pi} \frac{r^{n+1-2(j-k)} \cos ^{n+1-2(j-k)} \theta}{(r \cos \theta-\mathrm{a})(r \sin \theta-\mathrm{b})} d \theta .
\end{aligned}
$$

When $k<j$ the statement is proved using the induction hypothesis in each of the terms of the sum since, after the multiplication by the monomial $\left(r^{2}\right)^{j-k}$, we have that the polynomials $U\left(r^{2}\right), V\left(r^{2}\right)$ and $W\left(r^{2}\right)$ have degree $[(n-2(j-k)) / 2]+1+(j-k)=[n / 2]+1$, $[(n-2(j-k)) / 2]+1+(j-k)=[n / 2]+1$ and $[(n-2(j-k)-1) / 2]+(j-k)=[(n-1) / 2]$, respectively.

The unique case that remains to check is $k=j$ for both integrals:

$$
\int_{0}^{2 \pi} \frac{r^{n+1} \sin \theta \cos ^{n} \theta}{(r \cos \theta-a)(r \sin \theta-b)} d \theta \quad \text { and } \quad \int_{0}^{2 \pi} \frac{r^{n+1} \cos ^{n+1} \theta}{(r \cos \theta-a)(r \sin \theta-b)} d \theta \text {. }
$$

For the first one we can write

$$
\begin{aligned}
& \int_{0}^{2 \pi} \frac{r^{n+1} \sin \theta \cos ^{n} \theta}{(r \cos \theta-a)(r \sin \theta-b)} d \theta=\int_{0}^{2 \pi} \frac{r^{n}(r \sin \theta-b+b) \cos ^{n} \theta}{(r \cos \theta-a)(r \sin \theta-b)} d \theta= \\
& \int_{0}^{2 \pi} \frac{r^{n} \cos ^{n} \theta}{r \cos \theta-a} d \theta+b \int_{0}^{2 \pi} \frac{r^{n} \cos ^{n} \theta}{(r \cos \theta-a)(r \sin \theta-b)} d \theta .
\end{aligned}
$$

Thus we can take only the first term in the sum because for the second one we can apply once more the induction hypothesis. For this term, using the computation of $I_{n}^{\mathrm{a}}(r)$, the corresponding polynomial $W\left(r^{2}\right)$, that is called $T$ in Lemma 2.1, has degree $[(n-1) / 2]$ and the polynomial $U\left(r^{2}\right)$ can be written as $\left(\mathrm{a}^{2}+\mathrm{b}^{2}-r^{2}\right) S_{[(n-2) / 2]+1}\left(r^{2}\right)$ and it has degree $([(n-2) / 2]+1)+1=[n / 2]+1$. 
Now we will consider the last integral, that is

$$
\begin{aligned}
& \int_{0}^{2 \pi} \frac{r^{n+1} \cos ^{n+1} \theta}{(r \cos \theta-\mathrm{a})(r \sin \theta-\mathrm{b})} d \theta=\int_{0}^{2 \pi} \frac{r^{n} \cos ^{n} \theta(r \cos \theta-\mathrm{a}+\mathrm{a})}{(r \cos \theta-\mathrm{a})(r \sin \theta-\mathrm{b})} d \theta= \\
& \int_{0}^{2 \pi} \frac{r^{n} \cos ^{n} \theta}{r \sin \theta-\mathrm{b}} d \theta+\mathrm{a} \int_{0}^{2 \pi} \frac{r^{n} \cos ^{n} \theta}{(r \cos \theta-\mathrm{a})(r \sin \theta-\mathrm{b})} d \theta .
\end{aligned}
$$

Changing $\theta$ by $\theta+\pi / 2$ we can use the expression for $I_{n}^{\mathrm{b}}(r)$ computed in Lemma 2.1 to prove that, as in the previous computation, this integral satisfies the formula of the statement. The second term follows applying the induction hypothesis. Then the statement is proved.

Proposition 2.3. Let $K$ be a natural number, let $P_{n}(x, y)$ and $Q_{n}(x, y)$ be real polynomials of degree $n, \gamma_{r}=\left\{(x, y) \mid x^{2}+y^{2}=r^{2}\right\},\left\{\mathrm{a}_{1}, \ldots, \mathrm{a}_{K}\right\}$ different real numbers and

$$
I(r)=\int_{\gamma_{r}} \frac{Q_{n}(x, y) d x-P_{n}(x, y) d y}{\prod_{j=1}^{K}\left(x-\mathrm{a}_{j}\right)} .
$$

Then,

$$
I(r)=\sum_{j=1}^{\widetilde{K}} \frac{S_{[(n-1) / 2]+1}^{j}\left(r^{2}\right)}{\sqrt{\widetilde{\mathrm{a}}_{j}^{2}-r^{2}}}+T_{[n / 2]}\left(r^{2}\right),
$$

for suitable polynomials $S_{s}^{j}(\rho), T_{s}(\rho)$ of degree $s$, where $\widetilde{K}=\operatorname{Card}\left\{\left|\mathrm{a}_{1}\right|, \ldots,\left|\mathrm{a}_{K}\right|\right\}$ and $\widetilde{\mathrm{a}}_{1}, \ldots, \widetilde{\mathrm{a}}_{\widetilde{K}}$ denoting the different values of the set $\left\{\left|\mathrm{a}_{1}\right|, \ldots,\left|\mathrm{a}_{K}\right|\right\}$.

Proof. Parametrising $\gamma_{r}$ using polar coordinates, $(x, y)=(r \cos \theta, r \sin \theta)$, we can write

$$
I(r)=\int_{0}^{2 \pi} \frac{R_{n+1}(r \cos \theta, r \sin \theta)}{\prod_{j=1}^{K}\left(r \cos \theta-\mathrm{a}_{j}\right)} d \theta
$$

where $R_{n+1}(r \cos \theta, r \sin \theta)=-r Q_{n}(r \cos \theta, r \sin \theta) \sin \theta-r P_{n}(r \cos \theta, r \sin \theta) \cos \theta$. Performing partial fraction decomposition and using Lemma 2.1, it turns out that

$$
\begin{aligned}
I(r)= & \sum_{j=1}^{K} \int_{0}^{2 \pi} \frac{1}{\prod_{\substack{\ell=1 \\
\ell \neq j}}^{K}\left(\mathrm{a}_{\ell}-\mathrm{a}_{j}\right)} \frac{R_{n+1}(r \cos \theta, r \sin \theta)}{r \cos \theta-\mathrm{a}_{j}} d \theta= \\
& \sum_{j=1}^{K}\left(\frac{S_{[(n-1) / 2]+1}^{j}\left(r^{2}\right)}{\sqrt{\mathrm{a}_{j}^{2}-r^{2}}}+T_{[n / 2]}^{j}\left(r^{2}\right)\right)=\sum_{j=1}^{K}\left(\frac{S_{[(n-1) / 2]+1}^{j}\left(r^{2}\right)}{\sqrt{\mathrm{a}_{j}^{2}-r^{2}}}\right)+T_{[n / 2]}\left(r^{2}\right),
\end{aligned}
$$

provided we define $T_{[n / 2]}(\rho)=\sum_{j=1}^{K} T_{[n / 2]}^{j}(\rho)$. Since this expression depends only on the absolute values $\left|\mathrm{a}_{j}\right|$ we collect terms, consider new polynomials $S_{s}^{j}(\rho)$ and, at the end, get formula (7). 
Proposition 2.4. Let $K_{1}, K_{2}$ be natural numbers, $P_{n}(x, y)$ and $Q_{n}(x, y)$ be real polynomials of degree $n, \gamma_{r}=\left\{(x, y) \mid x^{2}+y^{2}=r^{2}\right\}$ and $\left\{\mathrm{a}_{1}, \ldots, \mathrm{a}_{K_{1}}, \mathrm{~b}_{1}, \ldots, \mathrm{b}_{K_{2}}\right\}$ real numbers satisfying that $\mathrm{a}_{j} \neq \mathrm{a}_{\ell}$ and $\mathrm{b}_{j} \neq \mathrm{b}_{\ell}$ for $j \neq \ell$. Consider

$$
I(r)=\int_{\gamma_{r}} \frac{Q_{n}(x, y) d x-P_{n}(x, y) d y}{\prod_{j=1}^{K_{1}}\left(x-\mathrm{a}_{j}\right) \prod_{k=1}^{K_{2}}\left(y-\mathrm{b}_{k}\right)} .
$$

Then,

$$
\begin{aligned}
I(r)=\sum_{j=1}^{K_{1}}\left(\sum_{k=1}^{K_{2}} \frac{U_{[n / 2]+1}^{j, k}\left(r^{2}\right)}{\mathrm{a}_{j}^{2}+\mathrm{b}_{k}^{2}-r^{2}}\right) & \frac{1}{\sqrt{\mathrm{a}_{j}^{2}-r^{2}}}+ \\
& \sum_{k=1}^{K_{2}}\left(\sum_{j=1}^{K_{1}} \frac{V_{[n / 2]+1}^{j, k}\left(r^{2}\right)}{\mathrm{a}_{j}^{2}+\mathrm{b}_{k}^{2}-r^{2}}\right) \frac{1}{\sqrt{\mathrm{b}_{k}^{2}-r^{2}}}+W_{[(n-1) / 2]}\left(r^{2}\right),
\end{aligned}
$$

for suitable polynomials $U_{s}^{j, k}(\rho), V_{s}^{j, k}(\rho)$ and $W_{s}(\rho)$ of degree $s$.

Proof. Parameterising $\gamma_{r}$ using polar coordinates, $(x, y)=(r \cos \theta, r \sin \theta)$, we can write

$$
I(r)=\int_{0}^{2 \pi} \frac{R_{n+1}(r \cos \theta, r \sin \theta)}{\prod_{j=1}^{K_{1}}\left(r \cos \theta-\mathrm{a}_{j}\right) \prod_{k=1}^{K_{2}}\left(r \sin \theta-\mathrm{b}_{k}\right)} d \theta
$$

where $R_{n+1}(r \cos \theta, r \sin \theta)=-r Q_{n}(r \cos \theta, r \sin \theta) \sin \theta-r P_{n}(r \cos \theta, r \sin \theta) \cos \theta$. Performing a partial fraction expansion and using Lemma 2.2 it follows that

$$
\begin{aligned}
I(r)= & \sum_{j=1}^{K_{1}} \sum_{k=1}^{K_{2}} \int_{0}^{2 \pi} \frac{1}{\prod_{\substack{\ell=1 \\
\ell \neq j}}^{K_{1}}\left(\mathrm{a}_{\ell}-\mathrm{a}_{j}\right)} \frac{1}{\prod_{\substack{\ell=1 \\
\ell \neq k}}^{K_{2}}\left(\mathrm{~b}_{\ell}-\mathrm{b}_{k}\right)} \frac{R_{n+1}(r \cos \theta, r \sin \theta)}{\left(r \cos \theta-\mathrm{a}_{j}\right)\left(r \sin \theta-\mathrm{b}_{k}\right)} d \theta= \\
& \sum_{j=1}^{K_{1}} \sum_{k=1}^{K_{2}}\left(\frac{1}{\mathrm{a}_{j}^{2}+\mathrm{b}_{k}^{2}-r^{2}}\left(\frac{U_{[n / 2]+1}^{j, k}\left(r^{2}\right)}{\sqrt{\mathrm{a}_{j}^{2}-r^{2}}}+\frac{V_{[n / 2]+1}^{j, k}\left(r^{2}\right)}{\sqrt{\mathrm{b}_{k}^{2}-r^{2}}}\right)+W_{[(n-1) / 2]}^{j, k}\left(r^{2}\right)\right)= \\
& \sum_{j=1}^{K_{1}}\left(\sum_{k=1}^{K_{2}} \frac{U_{[n / 2]+1}^{j, k}\left(r^{2}\right)}{\mathrm{a}_{j}^{2}+\mathrm{b}_{k}^{2}-r^{2}}\right) \frac{1}{\sqrt{\mathrm{a}_{j}^{2}-r^{2}}}+\sum_{k=1}^{K_{2}}\left(\sum_{j=1}^{K_{1}} \frac{V_{[n / 2]+1}^{j, k}\left(r^{2}\right)}{\mathrm{a}_{j}^{2}+\mathrm{b}_{k}^{2}-r^{2}}\right) \frac{1}{\sqrt{\mathrm{b}_{k}^{2}-r^{2}}}+ \\
& \sum_{j=1}^{K_{1}} \sum_{k=1}^{K_{2}} W_{[(n-1) / 2]}^{j, k}\left(r^{2}\right),
\end{aligned}
$$

for a collection of polynomials $U_{s}^{j, k}(\rho), V_{s}^{j, k}(\rho), W_{s}^{j, k}(\rho)$ of degree $s$ for all $j=1, \ldots, K_{1}$ and $k=1, \ldots, K_{2}$. Denoting $W_{[(n-1) / 2]}\left(r^{2}\right)=\sum_{j=1}^{K_{1}} \sum_{k=1}^{K_{2}} W_{[(n-1) / 2]}^{j, k}\left(r^{2}\right)$, the claimed result follows. 


\section{Proof of Theorem 1.2}

The proof of Theorem 1.2 will use the known as Derivation-Division procedure (see for instance [11, p. 119]). First we give two technical results useful to compute successive derivatives that appear applying this procedure to the function (5). The proof of the first one is very simple and implies that, in general,

$$
\mathcal{D}^{k}\left(p_{n}(x)\left(\frac{x+a}{x+b}\right)^{\alpha}\right)=q_{n+k}(x) \frac{(x+a)^{\alpha-k}}{(x+b)^{\alpha+k}} .
$$

The proof of the second one is a little more involved and, as we will see, it constitutes the key point for proving Theorem 1.2. It shows that the case $k=n+1$ in (8) is very special, since it undergoes a sudden drop of the degree of the polynomial $q_{n+k}(x)$.

Lemma 3.1. Consider $a \neq b$ and $\alpha \notin \mathbb{Z}$ real numbers. Then

$$
\mathcal{D}\left(p_{n}(x)(x+a)^{\alpha}\right)=q_{n}(x)(x+a)^{\alpha-1}, \quad \mathcal{D}\left(p_{n}(x)\left(\frac{x+a}{x+b}\right)^{\alpha}\right)=q_{n+1}(x) \frac{(x+a)^{\alpha-1}}{(x+b)^{\alpha+1}}
$$

where $p_{i}(x)$ and $q_{i}(x)$ are polynomials of degree $i$.

Lemma 3.2. Consider $a \neq b$ and $\alpha \notin \mathbb{Z}$ real numbers. Then

$$
\mathcal{D}^{n+1}\left(p_{n}(x)\left(\frac{x+a}{x+b}\right)^{\alpha}\right)=q_{n}(x) \frac{(x+a)^{\alpha-(n+1)}}{(x+b)^{\alpha+(n+1)}}
$$

where $p_{n}(x)$ and $q_{n}(x)$ are polynomials of degree $n$.

Proof. We will prove the statement inductively. It is not restrictive to consider $p_{n}(x)$ as a monic polynomial and written as $p_{n}(x)=(x+a)^{n}+p_{n-1}(x)$ for a suitable polynomial $p_{n-1}(x)$ of degree $n-1$. From the equalities

$$
\begin{aligned}
& \mathcal{D}^{n+1}\left(p_{n}(x)\left(\frac{x+a}{x+b}\right)^{\alpha}\right)=\mathcal{D}^{n+1}\left(\left((x+a)^{n}+p_{n-1}(x)\right)\left(\frac{x+a}{x+b}\right)^{\alpha}\right)= \\
& \mathcal{D}^{n+1}\left((x+a)^{n}\left(\frac{x+a}{x+b}\right)^{\alpha}\right)+\mathcal{D}\left(\mathcal{D}^{n}\left(p_{n-1}(x)\left(\frac{x+a}{x+b}\right)^{\alpha}\right)\right)
\end{aligned}
$$

and using Lemma 3.1, we have that it is enough to prove the statement for $p_{n}(x)=$ $(x+a)^{n}$.

Indeed, it is not difficult to check, inductively, that the following expression is satisfied

$$
\mathcal{D}^{j}\left(\frac{(x+a)^{\alpha+n}}{(x+b)^{\alpha}}\right)=\frac{(x+a)^{\alpha+n-j}}{(x+b)^{\alpha+j}} \sum_{\ell=0}^{j} C_{j, \ell}(x+a)^{\ell}
$$

with

$$
C_{j, \ell}=(-1)^{j+\ell}(a-b)^{j-\ell}\left(\begin{array}{l}
j \\
\ell
\end{array}\right) \frac{\Gamma(\alpha+n+1)}{\Gamma(\alpha+n-(j-\ell)+1)} \prod_{m=1}^{\ell}(n-j+m)
$$


where we define $\prod_{m=1}^{0}(n-j+m)=1$ and $\Gamma$ is the Gamma function. Therefore,

$$
\begin{gathered}
\mathcal{D}^{n+1}\left((x+a)^{n}\left(\frac{x+a}{x+b}\right)^{\alpha}\right)=\frac{(x+a)^{\alpha+n-(n+1)}}{(x+b)^{\alpha+(n+1)}} \sum_{\ell=0}^{(n+1)} C_{n+1, \ell}(x+a)^{\ell}= \\
\frac{(x+a)^{\alpha-1}}{(x+b)^{\alpha+n+1}}\left((-1)^{n+1}(a-b)^{n+1} \frac{\Gamma(\alpha+n+1)}{\Gamma(\alpha)}+\right. \\
\left.\sum_{\ell=1}^{n+1}(-1)^{n+1+\ell}(a-b)^{n+1-\ell}\left(\begin{array}{c}
n+1 \\
\ell
\end{array}\right) \frac{\Gamma(\alpha+n+1)}{\Gamma(\alpha+\ell)} \prod_{m=1}^{\ell}(m-1)\right) .
\end{gathered}
$$

In the latter expression, the terms corresponding to $\ell=1, \ldots, n+1$, clearly vanish and then it reduces to

$$
(-1)^{n+1}(a-b)^{n+1} \frac{\Gamma(\alpha+n+1)}{\Gamma(\alpha)}(x+a)^{n} \frac{(x+a)^{\alpha-(n+1)}}{(x+b)^{\alpha+n+1}} .
$$

Hence the statement is proved.

Proof of Theorem 1.2. Set $n_{0}=\operatorname{deg}\left(P^{0}\right)$. Differentiating $F(x)$ in $(5) n_{0}+1$ times, applying Lemma 3.1 and dividing by $\left(x+c_{1}\right)^{-\frac{1}{2}-\left(n_{0}+1\right)}$ we have

$$
F_{1}(x)=P_{n}^{1,1}(x)+\sum_{j=2}^{K} P_{n}^{j, 1}(x)\left(\frac{x+c_{j}}{x+c_{1}}\right)^{\alpha_{1}},
$$

where $P_{n}^{j, 1}(x)$ are suitable polynomials of degree at most $n=\max \left(\operatorname{deg} P^{1}, \ldots, \operatorname{deg} P^{K}\right)$ and $\alpha_{1}=-\frac{1}{2}-\left(n_{0}+1\right)$. Differentiating $F_{1}(x), n+1$ times, applying Lemma 3.2 and dividing by $\left(x+c_{2}\right)^{\alpha_{1}-(n+1)} /\left(x+c_{1}\right)^{\alpha_{1}+(n+1)}$ we have

$$
F_{2}(x)=P_{n}^{2,2}(x)+\sum_{j=3}^{K} P_{n}^{j, 2}(x)\left(\frac{x+c_{j}}{x+c_{2}}\right)^{\alpha_{2}},
$$

with $P_{n}^{2, j}(x)$ polynomials of degree $n$ and $\alpha_{2}=\alpha_{1}-(n+1)$. Performing the same procedure $K-2$ times we reach

$$
F_{K}(x)=P_{n}^{K, K}(x) .
$$

Note that all the polynomials $P_{n}^{j, \ell}(x)$ appearing in the process have degree $n$. Since the total number of derivatives is $(K-1)(n+1)+n_{0}+1$ and the degree of the last polynomial is $n$ the total number of zeroes of $F(x)$ is bounded by $K(n+1)+n_{0}$, as we wanted to prove.

\section{Proof of Theorem 1.1}

We start studying the case $K_{2}=0$. From the expression of Proposition 2.3 and applying Theorem 1.2 we know that the number of zeroes of $I(r)$ is less or equal than

$$
\widetilde{K}_{1}\left(\left[\frac{n-1}{2}\right]+2\right)+\left[\frac{n}{2}\right]=\widetilde{K}_{1}\left[\frac{n+3}{2}\right]+\left[\frac{n}{2}\right] .
$$


as we wanted to see.

Consider now $K_{2} \geq 1$. We know that the set $\left\{\mathrm{a}_{j}^{2}+\mathrm{b}_{\ell}^{2}, j=1, \ldots, K_{1}, \ell=1, \ldots, K_{2}\right\}$ has $L$ elements. Define the function

$$
H\left(r^{2}\right):=\prod_{i=1}^{L}\left(\mathrm{a}_{j_{i}}^{2}+\mathrm{b}_{\ell_{i}}^{2}-r^{2}\right),
$$

using all the different elements of the set. From Proposition 2.4 and multiplying $I(r)$ by $H\left(r^{2}\right)$ we obtain that

$$
\begin{aligned}
H\left(r^{2}\right) I(r)= & \sum_{j=1}^{K_{1}}\left(\sum_{k=1}^{K_{2}} \frac{H\left(r^{2}\right)}{\mathrm{a}_{j}^{2}+\mathrm{b}_{k}^{2}-r^{2}} U_{[n / 2]+1}^{j, k}\left(r^{2}\right)\right) \frac{1}{\sqrt{\mathrm{a}_{j}^{2}-r^{2}}}+ \\
& \sum_{k=1}^{K_{2}}\left(\sum_{j=1}^{K_{1}} \frac{H\left(r^{2}\right)}{\mathrm{a}_{j}^{2}+\mathrm{b}_{k}^{2}-r^{2}} V_{[n / 2]+1}^{j, k}\left(r^{2}\right)\right) \frac{1}{\sqrt{\mathrm{b}_{k}^{2}-r^{2}}}+H\left(r^{2}\right) W_{[(n-1) / 2]}\left(r^{2}\right)= \\
& \sum_{j=1}^{K_{1}} \widehat{U}_{[n / 2]+L}^{j}\left(r^{2}\right) \frac{1}{\sqrt{\mathrm{a}_{j}^{2}-r^{2}}}+\sum_{k=1}^{K_{2}} \widehat{V}_{[n / 2]+L}^{k}\left(r^{2}\right) \frac{1}{\sqrt{\mathrm{b}_{k}^{2}-r^{2}}}+\widehat{W}_{[(n-1) / 2]+L}\left(r^{2}\right)= \\
& \sum_{j=1}^{\widetilde{K}_{1}} \widehat{S}_{[n / 2]+L}^{j}\left(r^{2}\right) \frac{1}{\sqrt{\widetilde{\mathrm{a}}_{j}^{2}-r^{2}}}+\sum_{k=1}^{\widetilde{K}_{2}} \widehat{T}_{[n / 2]+L}^{k}\left(r^{2}\right) \frac{1}{\sqrt{\widetilde{\mathrm{b}}_{k}^{2}-r^{2}}}+\widehat{W}_{[(n-1) / 2]+L}\left(r^{2}\right),
\end{aligned}
$$

where the polynomials $\widehat{S}_{m}^{j}\left(r^{2}\right), \widehat{T}_{m}^{k}\left(r^{2}\right), \widehat{U}_{m}^{j}\left(r^{2}\right), \widehat{V}_{m}^{k}\left(r^{2}\right)$ and $\widehat{W}_{m}\left(r^{2}\right)$ have degree $m$ in $r^{2}$. In the last step $\widetilde{\mathrm{a}}_{1}, \ldots, \widetilde{\mathrm{a}}_{\widetilde{K}_{1}}$, denote the different values of the set $\left\{\left|\mathrm{a}_{1}\right|, \ldots,\left|\mathrm{a}_{K_{1}}\right|\right\}$ and $\widetilde{b}_{1}, \ldots, \widetilde{b}_{\widetilde{K}_{2}}$, denote the different values of the set $\left\{\left|b_{1}\right|, \ldots,\left|b_{K_{2}}\right|\right\} \backslash\left\{\left|a_{1}\right|, \ldots,\left|a_{K_{1}}\right|\right\}$. Remember that

$$
\widetilde{K}_{1}=\operatorname{Card}\left\{\left|\mathrm{a}_{1}\right|, \ldots,\left|\mathrm{a}_{K_{1}}\right|\right\} \quad \text { and } \quad \widetilde{K}_{2}=\operatorname{Card}\left\{\left|\mathrm{a}_{1}\right|, \ldots,\left|\mathrm{a}_{K_{1}}\right|,\left|\mathrm{b}_{1}\right|, \ldots,\left|\mathrm{b}_{K_{2}}\right|\right\}-\widetilde{K}_{1} .
$$

Applying Theorem 1.2 to the right hand part of the last equality we have the expression of the statement because the number of zeroes of $I(r)$ in $\left(0, \min \left\{\left|a_{j}\right|,\left|b_{\ell}\right|\right\}\right)$ satisfies

$$
\mathcal{Z}(I) \leq\left(\widetilde{K}_{1}+\widetilde{K}_{2}\right)([n / 2]+L+1)+[(n-1) / 2]+L
$$

Remark 4.1. The upper bounds given in Theorem 1.1 are maximal when all the straight lines are "generically" located, that is, when $\widetilde{K}_{1}=K_{1}, \widetilde{K}_{2}=K_{2}$ and $L=K_{1} K_{2}$ hold.

Denote by $K$ the total number of straight lines (parallel or orthogonal one-to-one) that we consider in our system. Then $K=K_{1}+K_{2}$. Therefore, the maximal value for the upper bound for the number of zeroes of the Abelian integral $I(r)$ is achieved for $K_{1}=K_{2}=K / 2$ if $K$ even and $K_{1}=(K+1) / 2, K_{2}=(K-1) / 2$ if $K$ odd. We have the following result:

Corollary 4.2. Under the hypotheses of Theorem 1.1 and denoting by $K=K_{1}+K_{2}$ the total of number of straight lines of singular points, we have that the number of zeroes 
of $I(r)$ satisfies

$$
\mathcal{Z}(I) \leq K\left[\frac{n}{2}\right]+\left[\frac{n-1}{2}\right]+(K+1)\left[\frac{K}{2}\right]\left[\frac{K+1}{2}\right]+K .
$$

Although in this work we do not consider the problem of the sharpness of the upper bounds for the Abelian integral $I(r)$ given in Theorem 1.1 we end this section with several comments about this question. Fixed some $K_{1}$ and $K_{2}$, first let us count how many effective free parameters gives the perturbation $(P, Q)$ in the expression of $I(r)$. In principle these two polynomials of degree $n$ have both together $(n+2)(n+1)$ parameters, but some symmetry considerations based on the first expression of $I(r)$, reduce this number in the computation of $I(r)$ to $(n+4)(n+1) / 2$ when $K_{1} K_{2} \neq 0$ and to $[(n+$ $5)(n+1) / 4$ ] when $K_{1} K_{2}=0$. On the other hand the growth of the upper bound for $\mathcal{Z}(I)$ is linear in $n$. This fact makes natural to believe that, fixed $K_{1}$ and $K_{2}$, the upper bounds for $n$ large enough are essentially reached.

Besides, when we restrict our study to families of polynomial vector fields of degree $n$ it is natural to take $K=n-1$. Thus, applying the previous results, the upper bound for the number of zeroes of the corresponding Abelian integral $I(r)$ grows as $n^{3} / 4$, when $K_{1} K_{2} \neq 0$, while the number of free parameters only grows as $n^{2} / 2$. This difference in growth implies, for $n$ large enough, the existence of a collection of relations between the coefficients of the functions $P^{j}(x)$ that appear when we apply Theorem 1.2 to the final expression of $I(r)$. Therefore, in this situation, it can not be expected at all the sharpness of the upper bound. When $K_{1} K_{2}=0$ something similar happens.

\section{Relations With SOME PREVIOUS WORKS}

There are some previous works where the results of this paper can be applied. In some cases we can give an alternative proof of the corresponding upper bound; in the other cases the upper bound is improved. We remark that in some of these works the sharpness of the obtained upper bounds is also studied. In our paper, this question has not been considered.

In [10] the authors deal with the case of a single straight line of critical points, $G(x, y)=x-1$. In this case they proved that $\mathcal{Z}(I) \leq n$ and that the bound is sharp. Our result gives an alternative proof for the upper bound.

The case with two straight lines of singularities was studied in [13] and [2]. The first one considered the case of both lines been parallel and the latter when they are perpendicular. In the first paper the authors proved that the number of zeroes satisfies

$$
\mathcal{Z}(I) \leq \begin{cases}2 n+3, & n \text { is odd } \\ 2 n+1, & n \text { is even, }\end{cases}
$$

while the upper bound provided by Theorem 1.1 is sharper:

$$
\mathcal{Z}(I) \leq \begin{cases}\frac{3 n+5}{2}, & n \text { is odd } \\ \frac{3 n+4}{2}, & n \text { is even }\end{cases}
$$


In the second paper, it was proved that

$$
\begin{cases}\frac{3 n+1}{2}, & n \text { is odd }, \\ \frac{3 n-2}{2}, & n \text { is even, }\end{cases}
$$

Using Theorem 1.1 we get that

$$
\mathcal{Z}(I) \leq \begin{cases}\frac{3 n+7}{2}, & n \text { is odd } \\ \frac{3 n+8}{2}, & n \text { is even. }\end{cases}
$$

The difference can be explained because the perturbation in [2] satisfies $P_{n}(0,0)=$ $Q_{n}(0,0)=0$ while our study has not this restriction.

In [5] the authors considered three lines, two parallel and one perpendicular. They proved that

$$
\left\{\begin{array}{ll}
2 n+2, & n \text { is odd }, \\
2 n, & n \text { is even, }
\end{array} \leq \mathcal{Z}(I) \leq \begin{cases}\frac{5 n+23}{2}, & n \text { is odd } \\
\frac{5 n+18}{2}, & n \text { is even }\end{cases}\right.
$$

Using Theorem 1.1, with $\left(K_{1}, K_{2}\right)=(2,1)$, the last inequalities can be improved to

$$
\mathcal{Z}(I) \leq \begin{cases}2 n+12, & n \text { is odd } \\ 2 n+13, & n \text { is even }\end{cases}
$$

The special case with four lines given by $G(x, y)=\left(x^{2}-a^{2}\right)\left(y^{2}-b^{2}\right)$ was studied in [1]. When $a \neq b$ the authors showed that

$$
\mathcal{Z}(I) \leq \begin{cases}\frac{3 n+5}{2}, & n \text { is odd } \\ \frac{3 n+2}{2}, & n \text { is even, }\end{cases}
$$

and it was claimed that these bounds are sharp. The upper bound given by Theorem 1.1 increases in one unit the previous ones. In our notation $\mathrm{a}_{1}=-\mathrm{a}_{2}=a$ and $\mathrm{b}_{1}=-\mathrm{b}_{2}=b$, and so $K_{1}=K_{2}=2$ but $\widetilde{K}_{1}=\widetilde{K}_{2}=L=1$. In the generic case $\left(K_{1}=K_{2}=\widetilde{K}_{1}=\right.$ $\widetilde{K}_{2}=L=2$ ) we get an upper bound that grows like $5 n / 2$.

\section{REFERENCES}

[1] A. Atabaigi, N. Nyamoradi and H. R. Z. Zangeneh. The number of limit cycles of a quintic polynomial system. Computers and Mathematics with Applications, 57, 677-684, 2009.

[2] A. Buică and J. Llibre. Limit cycles of a perturbed cubic polynomial differential center. Chaos Solitons Fractals 32, 1059-1069, 2007.

[3] C. Christopher and C. Li. Limit cycles of differential equations. Advanced Courses in Mathematics. CRM Barcelona. Birkhäuser Verlag, Basel, 2007. viii+171 pp.

[4] B. Coll, A. Gasull and R. Prohens. Bifurcation of limit cycles from two families of centers. Dyn. Contin. Discrete Impuls. Syst. Ser. A Math. Anal. 12, 275-287, 2005.

[5] B. Coll, J. Llibre and R. Prohens. Limit cycles bifurcating from a perturbed quartic center. Preprint, 2010.

[6] A. Gasull, J. T. Lázaro and J. Torregrosa. A new family of Chebyshev systems. In preparation, 2011.

[7] A. Gasull, R. Prohens and J. Torregrosa. Bifurcation of limit cycles from a polynomial non-global center. J. Dyn. Diff. Equat. 20, 945-960, 2008.

[8] J. Giné and J. Llibre. Limit cycles of cubic polynomial vector fields via the averaging theory. Nonlinear Anal. 66, number 8, 1707-1721, 2007. 
[9] C. Li, J. Llibre and Z. Zhang. Weak focus, limit cycles and bifurcations for bounded quadratic systems. J. Differential Equations 115, 193-223, 1995.

[10] J. Llibre, J.S. Pérez del Río and J.A. Rodríguez. Averaging analysis of a perturbed quadratic center. Nonlinear Anal. 46, 45-51, 2001.

[11] R. Roussarie. Bifurcation of planar vector fields and Hilbert's sixteenth problem. Progress in Mathematics, 164. Birkhäuser Verlag, Basel, 1998. xviii+204 pp.

[12] G. Xiang and M. Han. Global bifurcation of limit cycles in a family of multiparameter systems. Internat. J. Bifur. Chaos 14, 3325-3335, 2004.

[13] G. Xiang and M. Han. Global bifurcation of limit cycles in a family of polynomial systems. $J$. Math. Anal. Appl. 295, 633-644, 2004.

Departament de Matemàtiques, Universitat Autònoma de Barcelona, 08193 BelLATERRA, BARCELONA, SPAin

E-mail address: gasull@mat.uab.cat

Departament de Matemàtica Aplicada i, Universitat Politècnica de Catalunya, Barcelona, SPAIN

E-mail address: jose.tomas.lazaro@upc.edu

Departament de Matemàtiques, Universitat Autònoma de Barcelona, 08193 BelLATERRA, BARCELONA, SPAIN

E-mail address: torre@mat.uab.cat 